\title{
BMJ Open Medical assessment of potential concussion in elite football: video analysis of the 2016 UEFA European championship
}

\author{
Karan Joshua Abraham, ${ }^{\oplus 1}$ Julia Casey, ${ }^{1}$ Arsenije Subotic, ${ }^{1}$ Christopher Tarzi, ${ }^{1}$ \\ Alice Zhu, ${ }^{1}$ Michael D Cusimano ${ }^{1,2}$
}

To cite: Abraham KJ, Casey J, Subotic A, et al. Medical assessment of potential concussion in elite football: video analysis of the 2016 UEFA European championship. BMJ Open 2019;9:e024607. doi:10.1136/ bmjopen-2018-024607

- Prepublication history for this paper is available online. To view these files, please visit the journal online (http://dx.doi org/10.1136/bmjopen-2018024607).

Received 11 June 2018 Revised 23 March 2019 Accepted 26 March 2019

Check for updates

(C) Author(s) (or their employer(s)) 2019. Re-use permitted under CC BY-NC. No commercial re-use. See rights and permissions. Published by BMJ.

${ }^{1}$ Neurosurgery, St. Michael's Hospital, Toronto, Ontario,

Canada

${ }^{2}$ Public Health, University of

Toronto Dalla Lana School of

Public Health, Toronto, Ontario, Canada

Correspondence to Dr Michael D Cusimano; injuryprevention@smh.ca

\section{ABSTRACT}

Objective The objective is to determine if suspected concussions in elite football are medically assessed according to the International Conferences on Concussion in Sport consensus statement recommendations.

Setting Men's Union of European Football Association (UEFA) Football Championship.

Participants All professional football players in the UEFA 2016 Championship Tournament.

Design Observational study.

Outcome measures Potential concussive events (PCEs) were defined as direct head collision incidents resulting in the athlete being unable to immediately resume play following impact. PCEs identified and description of PCE assessment and outcome were accomplished through direct standardised observation of video footage by trained observers in 51 games played in the Men's UEFA European Championship (10 June-10 July 2016).

Results Sixty-nine total PCEs (1.35 per match) were identified in 51 games played during the 2016 Men's UEFA European Championship. Forty-eight PCEs (69.6\%) resulted in two observable signs of concussion, 13 $(18.8 \%)$ resulted in three signs and $1(1.4 \%)$ resulted in four signs in the injured athletes. Nineteen (27.5\%) PCEs were medically assessed by sideline healthcare personnel while $50(72.5 \%)$ were not. Of the 50 PCEs that were not medically assessed, 44 (88\%) PCEs resulted in two or more signs of concussion among injured athletes. Of the 19 medically assessed PCEs, 8 resulted in 3 signs of concussion, and 1 resulted in 4 signs; all assessments concluded in the same-game return for the injured athletes.

Conclusions PCEs were frequent events in the 2016 UEFA Euro championship, but were rarely assessed concordant with the International Conferences on Concussion in Sport consensus statement recommendations. There is an imperative need to improve the assessment and management of players suspected of concussion in elite football.

\section{INTRODUCTION}

Sport-related concussion (SRC) is a frequent type of mild traumatic brain injury (mTBI) that has emerged as a major public health
Strengths and limitations of this study

- In this study, we sought to determine if suspected concussions in elite football are medically assessed according to the International Conferences on Concussion in Sport consensus statement recommendations to improve concussion awareness and safety measures in football-dominant nations.

- This study shows that there is an urgent need to improve the assessment and management of players suspected of concussion in the realm of elite football.

- The major weakness of this study is that video analysis provides limited information on the details of medical evaluation for potential concussive events and thus cannot always provide information on whether or not an appropriate medical decision is made.

concern. In children, adolescents, and young adults, sports participation is recognised as one of the leading causes of concussion. ${ }^{1}$ The USA National Centers for Disease Control and Prevention reported that sports-related and recreation-related TBI was responsible for 3.4 million visits to emergency departments between 2001 and 2012; approximately $70 \%$ of reported cases involved persons 19 or younger. ${ }^{2}$ Accounting for unreported, unrecognised and untreated injuries, the total number of sports-related TBI per year was estimated to be as high as 1.8-3.8 million in the USA, the majority of them being mTBI. ${ }^{3}$ Although most individuals experiencing mTBI are likely to recover within 1-3 weeks, a sizeable minority (up to $30 \%$ in some studies) may suffer from persistent concussion symptoms (ie, postconcussion syndrome). ${ }^{4} 5$ Furthermore, $15 \%-25 \%$ of mTBI cases are associated with adverse long-term physical, cognitive, and emotional sequelae. ${ }^{1}$ The high incidence and economic costs of SRC, preferential risk among vulnerable youth and 
potential for adverse long-term consequences underscore the need to better prevent, identify and manage such injuries.

Athletes involved in football, also called 'soccer' in North America, consistently experience among the highest rates of concussion ${ }^{67}$ Football is the world's most popular and fastest growing sport; an estimated 270 million people play the sport in various professional, semiprofessional and/or organised formal recreational capacities. ${ }^{8}$ By virtue of its widespread popularity, football's cumulative contribution to total SRC is likely to significantly overshadow other sports. Moreover, concussion awareness and safety measures remain relatively underdeveloped in many football-dominant nations. ${ }^{9}$ Such conditions are conducive to large numbers of undiagnosed concussions, inappropriate management and increased risk of potentially severe neurological consequences among vulnerable and/or improperly rehabilitated football athletes.

The proper assessment of suspected concussions immediately following injury is an important practice needed for early diagnosis and safe rehabilitation of athletes with brain injuries. Consensus statement recommendations published by fourth and fifth International Conferences on Concussion in Sport in 2012 and 2016, respectively, provide clear recommendations for the assessment of suspected concussions during a competitive event. ${ }^{10} 11$ The Concussion in Sport Group (CISG) 2012 and 2016 consensus statements assert that when an athlete shows any features of a concussion, the athlete should be (1) evaluated by a physician or another licensed healthcare provider onsite, (2) assessed using Sport Concussion Assessment Tool 3/5 (SCAT3/SCAT5) or other sideline concussion assessment tools and (3) prevented from return to play (RTP) in the event of a positive diagnosis. ${ }^{10} 11$ These statements were previously accepted and endorsed by football's major international governing body the Fédération Internationale de Football Association (FIFA). However, our previous analysis of 2014 Men's FIFA world cup (WC) found that the majority of potential concussive events (PCEs) $(63 \%)$ were not medically assessed, indicating a lack of congruence between recommendations and current practices at the elite level. ${ }^{12}$ In this study, we used video analysis to characterise PCEs and their assessment in the 2016 Men's Union of European Football Association (UEFA) European Championship. Additionally, we compared our findings against our previous analysis of the 2014 FIFA WC to determine if compliance with CISG recommendations had improved over the 2-year gap between tournaments.

\section{METHODS}

Video analysis has emerged as a reliable and valid tool to assess concussion in a variety of sports including American football, mixed martial arts, ice hockey and lacrosse. ${ }^{12-15}$ Furthermore, information content in digital videos can be analysed systematically to yield key insights into situational factors and injury-prone patterns of play leading to an injury.

\section{Coding of events}

PCEs were defined as any event in which one or more athletes experienced a head impact injury (through direct contact with another athlete, ball or object in the environment) and were unable to immediately resume play following impact. Athletes involved in PCEs were observed for six established observable physical signs of concussion: slow to get up (defined as a duration of 5 or more seconds following impact), clutching of the head, disorientation, loss of consciousness, seizure-like behaviour and signs of obvious disequilibrium. ${ }^{12}{ }^{16}$ The term PCE is not used synonymously with SRC. Instead, it captures the broad range of in-game scenarios involving head collisions; recognising that the higher the number of concussion signs associated with a PCE, the greater the index for suspicion that it may represent a bona fide concussion. Information pertaining to the assessment of athletes sustaining a PCE, including the personnel assessing the players (medical personnel, other players, the referee, no one), was recorded. Trained observers also collected information on PCE outcomes: RTP after assessment on pitch, RTP after assessment on sidelines, removed for remainder of match and removed from tournament.

\section{Training of reviewers}

Four observers were trained using video footage of four Premier League football matches in order to correctly identify PCEs and collect information pertaining to several variables on a standardised form used in a previous study on concussion. ${ }^{15}$ The standardised data collection form was used to provide a person viewing digital video images with a consistent way of coding and accounting for the majority of circumstances and mechanisms leading to concussion. The standardised data form was adapted from a validated form used in a prior study on concussion. ${ }^{15}$ The form was accompanied by a detailed data dictionary outlining the codes associated with each variable.

\section{Video analysis}

The observers analysed footage of the 51 games that took place during the 2016 UEFA European Championship from 10 June to 10 July. Observers independently reviewed the video footage to complete the standardised form. Three observers watched all 51 games of Men's UEFA Euro 2016 tournament, identified PCEs and collected data on aforementioned variables. All variables yielded a Cohen's kappa value of 0.90 or greater between all reviewers. Following this analysis, discrepancies in data were resolved through consultation with a fourth independent observer.

\section{Statistical analysis}

Descriptive statistics were reported as counts or frequencies and their associated percentages. All statistical analysis was done in STATA V.13 (StataCorp, Texas, USA). 
Table 1 Potential concussive event (PCE) assessment and outcome at Euro 2016

\begin{tabular}{|c|c|c|c|c|c|}
\hline \multirow[b]{2}{*}{$\begin{array}{l}\text { No. of } \\
\text { concussion } \\
\text { signs }\end{array}$} & \multirow[b]{2}{*}{$\begin{array}{l}\text { No. of PCE } \\
\text { (\% total) }\end{array}$} & \multicolumn{2}{|c|}{ PCE assessment (n) } & \multicolumn{2}{|c|}{$\begin{array}{l}\text { RTP outcome when medically } \\
\text { assessed (n) }\end{array}$} \\
\hline & & No assessment & $\begin{array}{l}\text { Medical } \\
\text { assessment }\end{array}$ & Same-game RTP & $\begin{array}{l}\text { Removed } \\
\text { from game or } \\
\text { tournament }\end{array}$ \\
\hline 0 & $2(2.9)$ & 1 & 1 & 1 & 0 \\
\hline 1 & $5(7.2)$ & 5 & 0 & 0 & 0 \\
\hline 3 & $13(18.8)$ & 5 & 8 & 8 & 0 \\
\hline 4 & $1(1.4)$ & 0 & 1 & 1 & 0 \\
\hline Total (\%) & 69 (100) & $50(72.5)$ & $19(27.5)$ & $19(100)$ & $0(0)$ \\
\hline
\end{tabular}

RTP, return to play.

Patient and public involvement

Patients and/or public were not involved.

\section{RESULTS}

\section{PCE incidence at Euro 2016}

Sixty-nine PCEs involving 61 different athletes were identified over the course of 51 games played during the 2016 UEFA European Championship (1.35 per match). Seven incidents involved direct head collisions between two athletes, which were scored as individual PCEs for each athlete. Of the 61 athletes who experienced a PCE, 4 $(6.6 \%)$ sustained 2 separate PCEs, and $2(3.3 \%)$ sustained 3 separate PCEs over the course of the tournament. Of the 69 PCEs recorded, $2(2.9 \%)$ resulted in no observable physical signs of concussion, $5(7.2 \%)$ resulted in 1 sign, $48(69.6 \%)$ resulted in 2 signs, $13(18.8 \%)$ resulted in 3 signs and 1 resulted in 4 signs (1.4\%) (table 1).

\section{Medical assessment of PCEs at Euro 2016}

As illustrated in table 1, of the 69 PCE incidents, 19 $(27.5 \%)$ were medically assessed by sideline healthcare personnel while $50(72.4 \%)$ were not. Of the 50 PCEs that were not medically assessed, $9(18 \%)$ received some attention from the referee and/or another player, while $41(82 \%)$ did not; all athletes involved in these incidents remained on the field following the incident. Among the 50 PCE incidents that did not receive medical assessment,
1 (2\%) showed no physical signs of concussion, 5 (10\%) showed 1 sign, 39 (78\%) showed 2 signs and $5(6.4 \%)$ showed 3 signs of concussions. Therefore, 44 out of the $50(88 \%)$ non-assessed PCEs involved athletes who exhibited two or more signs of concussion.

Among the 19 PCE incidents that were medically assessed by healthcare professionals, $1(5.3 \%)$ showed no physical signs of concussion, 9 (47.3\%) showed 2 signs, $8(42.1 \%)$ showed 3 signs and $1(5.3 \%)$ showed 4. All $19(100 \%)$ medically assessed PCEs concluded with the athlete returning to play following a brief assessment; no athletes were removed from the game or for the remainder of the tournament.

\section{Medical assessment of concussion: FIFA WC 2014 versus UEFA Euros 2016}

Table 2 compares PCE assessment and outcome in Euros 2016 versus our data from our previously published PCE analysis of WC 2014. ${ }^{12}$ PCE incidence was higher in the Euros (1.35 per match) compared with FIFA WC 2014 (1.13 per match). In Euro 2016, 89.8\% of PCEs involved athletes with two or more signs of concussion compared with $82.7 \%$ for WC 2014. Medical assessment of PCE at Euro 2016 (27.5\% assessed, $72.5 \%$ not assessed) was worse in relation to FIFA WC 2014 (37\% assessed, 63\% not assessed). In WC 2014, of the 29 PCEs that were medically assessed and involved athletes with 2 or more signs of

Table 2 Comparison of potential concussive event (PCE) assessment and outcome: Union of European Football Association Euro 2016 versus Fédération Internationale de Football World

\begin{tabular}{|c|c|c|c|c|c|c|c|c|}
\hline \multirow{2}{*}{$\begin{array}{l}\text { No. of } \\
\text { concussion } \\
\text { signs }\end{array}$} & \multicolumn{2}{|c|}{ No. of PCE (\% of total) } & \multicolumn{2}{|c|}{ PCEs medically assessed (n) } & \multicolumn{2}{|c|}{$\begin{array}{l}\text { PCEs not medically } \\
\text { assessed (n) }\end{array}$} & \multicolumn{2}{|c|}{$\begin{array}{l}\text { Medically assessed PCEs } \\
\text { with the same-game RTP (n) }\end{array}$} \\
\hline & WC 2014 & Euro 2016 & WC 2014 & Euro 2016 & WC 2014 & Euro 2016 & WC 2014 & Euro 2016 \\
\hline 1 & $11(14)$ & $5(7.2)$ & 1 & 0 & 10 & 5 & 1 & 0 \\
\hline 2 or more & $67(82.7)$ & $62(89.8)$ & 29 & 18 & 38 & 44 & 27 & 18 \\
\hline Total (\%) & $81(100)$ & $69(100)$ & $30(37)$ & $19(27.5)$ & $51(63)$ & $50(72.5)$ & $28(93)$ & $19(100)$ \\
\hline
\end{tabular}

WC, world cup 
concussion, $27(93.1 \%)$ returned to the same game, while $2(6.9 \%)$ were removed from the game and/or tournament. In Euro 2016, of the 18 medically assessed PCEs involving athletes with 2 or more signs of concussion, 18 $(100 \%)$ resulted in the same-game RTP.

\section{DISCUSSION}

\section{Concussion in football-an important public health concern}

Concussion is an inherent risk associated with sports participation and SRC is now recognised as a major public health concern. ${ }^{17}$ Yet, participation in sports is a meaningful part of millions of people's lives, and provides numerous physical and emotional health benefits. Maximising these benefits while simultaneously minimising the risks of adverse events such as concussion and its long-term sequelae represents an important public health imperative. Athletes involved in contact and/or collision sports are at higher risk for SRC. Football is a sport with an underappreciated high rate and burden of concussion. ${ }^{167}$ By virtue of football's global dimension and contribution to worldwide sport-related mTBI, sustained efforts at improving concussion awareness and assessment at all levels of the sport will have a substantial impact on reducing disability from and/or risk of injury.

\section{Inadequate implementation of consensus statement recommendations}

Medical assessment of a PCE is an important practice needed for early concussion diagnosis, proper management and safe RTP for injured athletes. Successful implementation of medical assessment protocols in sport is imperative to the larger effort to reduce SRC morbidity and its potentially serious long-term consequences. To aid this effort, clear protocols for on-field assessment of an athlete with a suspected concussion were published and reiterated in consensus statements following the fourth and fifth International Conferences on Concussion in Sport held in 2012 and 2016, respectively. ${ }^{10} 11$ These endeavours have been supported and endorsed by football's major international governing body FIFA. ${ }^{10} 11$ The CISG recommendations assert that when an athlete shows any features of a concussion, the athlete should be (1) evaluated by a physician or another licensed healthcare provider onsite, (2) assessed using SCAT3/ SCAT5 or other sideline concussion assessment tools and (3) prevented from RTP in the event of a positive diagnosis. ${ }^{1011}$ In a previous study on the Men's 2014 FIFA WC, we found that $63 \%$ of elite-level football athletes who sustained PCEs were not medically assessed, thereby underscoring the need for better implementation of concussion assessment protocols in elite football. Our current analysis of the 2016 UEFA European Championship reinforces this notion since only $27.5 \%$ of PCEs were medically assessed while $72.5 \%$ were not. While many PCEs are unlikely to result in bona fide concussions, it is clear that CISG recommendations apply to PCEs in which the index of suspicion for concussion is high (eg,
PCEs with multiple signs of concussion). Notably, 44 out of the $50(88 \%)$ non-assessed PCEs in the 2016 UEFA European Championship involved athletes who exhibited 2 or more signs of concussion. A proper assessment for concussion is warranted in such situations and a failure to assess raises legitimate concerns that some concussions may be missed. Furthermore, all PCEs that were medically assessed culminated in the same-game RTP despite the majority of these incidents resulting in 2 or more physical signs among injured athletes. Notably, the only athlete who experienced four physical signs of concussion was allowed to return to game following assessment, while another athlete who suffered a PCE incident that resulted in a head laceration, heavy bleeding and two physical signs of concussion was allowed to return to the game and sustained a second PCE shortly after returning. Thus, our data also indicate that the quality and accuracy of assessment received by athletes suffering PCEs are in need of careful scrutiny. Injury reporting and analysis by UEFA and FIFA should include more detail on PCEs and PCE evaluation in order to identify opportunities to improve athlete safety. ${ }^{18}$ Overall, our data highlight an obvious need to improve concussion assessment in the realm of elite football, and suggest that significant and persistent knowledge uptake and/or attitudinal barriers to implementation may exist.

\section{Barriers to implementation}

Several factors may contribute to weak implementation of SRC assessment protocols in elite football. First, the high stakes of competitive sport and rules of the games may pressure team medical staff to ignore PCEs, or inappropriately shorten examination and encourage RTP, particularly in situations when the team needs a result. In a widely publicised and recent example illustrating the pressures that team doctors may experience, a long-serving female team doctor from Chelsea Football Club was publically criticised, demoted and eventually forced out of the club for fulfilling her medical obligation to assess an injured player in stoppage time of a Premier League game. The current rules of the game, including running time and limited substitutions, further discourage pulling player for medical assessment. For the recent 2018 WC competition, FIFA implemented a new protocol for head injuries and concussions. This includes giving the referee the ability to stop the match for $3 \mathrm{~min}$ if a head injury is suspected for an on-pitch assessment. $3 \mathrm{~min}$ is not sufficient time to complete a SCAT3/5. The SCAT5 explicitly states on the form 'The SCAT5 cannot be performed correctly in less than $10 \mathrm{~min}$ ' ${ }^{19}$ Second, factors that normally contribute to under-reporting in other sports may be relevant as implementation barriers in football. ${ }^{20}$ For instance, a culture of toughness and loyalty to the team may either openly or subtly encourage athletes to downplay concussion or inappropriately accelerate RTP following injury. ${ }^{20}$ Furthermore, athletes are influenced by a strong desire to win, and fear of letting their teams or nations down, which may also encourage under-reporting 
of SRC symptoms. Third, our data suggest that significant gaps in knowledge translation exist, and highlight the need for improved knowledge dissemination and uptake at multiple levels including international football associations such as FIFA and UEFA, individual franchises, as well as associated staff, coaches, athletes and the wider spectrum of stakeholders in the sport. Research identifying barriers to implementation such as knowledge translation bottlenecks followed by strategic targeting of such impediments will be imperative to improved implementation of risk-reducing practices (including but not limited to medical assessment) that ultimately safeguard the long-term brain health of football athletes. Fourth, there may be a misperception and/or lack of awareness of SRC risk among football athletes, coaches, franchises and related organisations, which could limit enforcement. Advances in the prevention and proper management of SRC require that sporting associations attend to concussion with the same diligence typically applied to doping and other serious breaches. Reaching out directly to stakeholders, including FIFA, national team coaches and their staff physicians, as well as the intended beneficiaries of this work (athletes) will be imperative to widespread knowledge dissemination and uptake efforts.

In order to increase effectiveness of current football concussion protocols, it would be beneficial for football governing bodies to examine how other professional sports deal with concussions. For example, in NFL American football, the latest concussion protocol published in 2018 stipulates that each team during game day must be assigned a sideline unaffiliated neurotrauma consultant ('sideline UNC'), a physician that is impartial and independent from any Club. ${ }^{22}$ Additionally, the protocol also stipulates that a video UNC be present in a stadium booth with access to multiple views of video. ${ }^{22}$ Having impartial evaluators would help significantly in curtailing issues such as doctors from home teams facing pressure to return players to the game and examining the nature of potential concussive impacts. Providing broadcast video to sideline medical personnel in real time is also a recommendation by a recently published study that examined current practices related to video review of concussion in professional sports internationally. ${ }^{23}$ Football authorities should consider implementing these rules which would help aid in the screening for concussion and treat it as soon as possible.

\section{Limitations}

Video analysis provides limited information on the details of medical evaluation for PCEs and thus cannot always provide information on whether or not an appropriate medical decision is made. In some PCEs, full description of variables may be limited by the camera angles available for the video clip. Some game events may be misidentified as PCEs if players simulate or feign injury. Finally, it is possible, though unlikely, that some PCEs do not result in stoppage of play and the athlete continues to play resulting in a PCE being erroneously excluded from analysis.

\section{Future directions}

Overall, our data indicate that PCEs occur relatively frequently (at least one per game) in elite level football matches, but are rarely assessed in a manner concordant with the 2012 and 2016 CISG recommendations. Major international tournaments such as the FIFA WC and UEFA European Championships feature elite-level athletes and are among the most widely broadcasted and followed sporting events on the planet. ${ }^{24}$ The norms that persist on such a stage are likely to influence attitudes towards concussion on a global scale. Future major international tournaments offer the opportunity to set a new precedent for excellence in enforcement of concussion assessment, and initiate beneficial improvements in concussion awareness and the implementation of proper assessment protocols in the sport.

Contributors Research concept and design by MDC; data collection and analysis by KJA, JC, AS, CT and AZ; manuscript writing by KJA, JC and MDC. Guarantor: KJA. MDC is a non-paid volunteer on the expert advisory committee of Parachute Canada a not-for-profit injury prevention organization. He is a neurosurgeon at St. Michael's hospital who wishes he would never see one more brain injured person and that we can prevent every brain injury in the future.

Funding This work is supported by the Canadian Institutes of Health Research Strategic Team Grant in Applied Injury Research \#TIR-103946 and the Ontario Neurotrauma Foundation.

Competing interests None declared.

Patient consent for publication Not required.

Provenance and peer review Not commissioned; externally peer reviewed.

Data sharing statement The authors are happy to share data published in this study but most if not all data are already included in the manuscript and/or in the public domain.

Open access This is an open access article distributed in accordance with the Creative Commons Attribution Non Commercial (CC BY-NC 4.0) license, which permits others to distribute, remix, adapt, build upon this work non-commercially, and license their derivative works on different terms, provided the original work is properly cited, appropriate credit is given, any changes made indicated, and the use is non-commercial. See: http://creativecommons.org/licenses/by-nc/4.0/.

\section{REFERENCES}

1. Mannix R, Meehan WP, Pascual-Leone A. Sports-related concussions - media, science and policy. Nat Rev Neurol 2016;12:486-90.

2. Coronado VG, Haileyesus T, Cheng TA, et al. Trends in Sports- and Recreation-Related Traumatic Brain Injuries Treated in US Emergency Departments: The National Electronic Injury Surveillance SystemAll Injury Program (NEISS-AIP) 2001-2012. J Head Trauma Rehabil 2015;30:185-97.

3. Langlois JA, Rutland-Brown W, Wald MM. The epidemiology and impact of traumatic brain injury: a brief overview. J Head Trauma Rehabil 2006;21:375-8.

4. Zemek R, Barrowman N, Freedman SB. Clinical risk score for persistent postconcussion symptoms among childrenwith acute concussion in the ED (vol 315, pg 1014, 2016). Jama-J Am Med Assoc 2016;315:2624-24.

5. Guskiewicz KM, Marshall SW, Bailes J, et al. Recurrent concussion and risk of depression in retired professional football players. Med Sci Sports Exerc 2007;39:903-9.

6. Covassin T, Swanik CB, Sachs ML. Sex differences and the incidence of concussions among collegiate athletes. J Athl Train 2003;38:238-44.

7. Agel J, Evans TA, Dick R, et al. Descriptive epidemiology of collegiate men's soccer injuries: National collegiate athletic association injury 
surveillance system, 1988-1989 through 2002-2003. J Athl Train 2007;42:270-7.

8. Kunz M. 265 million playing football. FIFA magazine 2007:10-15.

9. Broglio SP, Vagnozzi R, Sabin M, et al. Concussion occurrence and knowledge in Italian football (soccer). J Sport Sci Med 2010;9:418-30.

10. McCrory P, Meeuwisse WH, Aubry M, et al. Consensus statement on concussion in sport: the 4th International Conference on Concussion in Sport held in Zurich, November 2012. Br J Sports Med 2013;47:250-8.

11. McCrory P, Meeuwisse WH, Dvořák J, et al. 5th International Conference on Concussion in Sport (Berlin). Br J Sports Med 2017; $51: 837-37$

12. Cusimano MD, Casey J, Jing R, et al. Assessment of head collision events during the 2014 fifa world cup tournament. JAMA 2017;317:2548-9.

13. Makdissi M, Davis G. Using video analysis for concussion surveillance in Australian football. J Sci Med Sport 2016;19:958-63.

14. McCrea M, Guskiewicz KM, Marshall SW, et al. Acute effects and recovery time following concussion in collegiate football players: the NCAA Concussion Study. JAMA 2003;290:2556-63.

15. Lawrence DW, Hutchison MG, Cusimano MD, et al. Interrater agreement of an observational tool to code knockouts and technical knockouts in mixed martial arts. Clin J Sport Med 2014;24:397-402.
16. Makdissi M, Davis G. The reliability and validity of video analysis for the assessment of the clinical signs of concussion in Australian football. J Sci Med Sport 2016;19:859-63.

17. Jordan BD. The clinical spectrum of sport-related traumatic brain injury. Nat Rev Neurol 2013;9:222-30.

18. Group IS. UEFA Injury study report. 2016:15.

19. Purtzki J, Chan C. Sport concussion assessment tool - 5th edition. Br J Sports Med 2017;51:851-8.

20. Cusimano MD, Topolovec-Vranic J, Zhang S, et al. Factors Influencing the Underreporting of Concussion in Sports: A Qualitative Study of Minor Hockey Participants. Clin J Sport Med 2017;27:375-80.

21. Cusimano MD, Cho N, Amin K, et al. Mechanisms of Team-SportRelated Brain Injuries in Children 5 to 19 Years Old: Opportunities for Prevention. PLoS One 2013;8:ARTN e58868:e58868.

22. Ellenbogen RG, Batjer $\mathrm{H}$, Cardenas $\mathrm{J}$, et al. National football league head, neck and spine committee's concussion diagnosis and management protocol: 2017-18 season. Br J Sports Med 2018;52:894-902.

23. Davis GA, Makdissi M, Bloomfield P, et al. International study of video review of concussion in professional sports. Br J Sports Med 2018:doi: bjsports-2018-099727 (Published Online First: 29 Sep 2018).

24. FIFA. 2014 FIFA World CUp breaks online streaming records, 2014. 